\title{
Precision Calculation of Hyperfine Constants for Extracting Nuclear Moments of ${ }^{229} \mathrm{Th}$
}

\author{
S. G. Porsev $\oplus^{1,2}$ M. S. Safronova, ${ }^{1}$ and M. G. Kozlov $\oplus^{2,3}$ \\ ${ }^{1}$ Department of Physics and Astronomy, University of Delaware, Newark, Delaware 19716, USA \\ ${ }^{2}$ Petersburg Nuclear Physics Institute of NRC "Kurchatov Institute”, Gatchina, Leningrad District 188300, Russia \\ ${ }^{3}$ St. Petersburg Electrotechnical University LETI, St. Petersburg 197376, Russia
}

(Received 5 August 2021; accepted 29 November 2021; published 17 December 2021)

\begin{abstract}
Determination of nuclear moments for many nuclei relies on the computation of hyperfine constants, with theoretical uncertainties directly affecting the resulting uncertainties of the nuclear moments. In this work, we improve the precision of such a method by including for the first time an iterative solution of equations for the core triple cluster amplitudes into the relativistic coupled-cluster method, with large-scale complete basis sets. We carried out calculations of the energies and magnetic dipole and electric quadrupole hyperfine structure constants for the low-lying states of ${ }^{229} \mathrm{Th}^{3+}$ in the framework of such a relativistic coupled-cluster single double triple method. We present a detailed study of various corrections to all calculated properties. Using the theory results and experimental data, we found the nuclear magnetic dipole and electric quadrupole moments to be $\mu=0.366(6) \mu_{N}$ and $Q=3.11(2) e b$, respectively, and reduce the uncertainty of the quadrupole moment by a factor of 3. The Bohr-Weisskopf effect of the finite nuclear magnetization is investigated, with bounds placed on the deviation of the magnetization distribution from the uniform one.
\end{abstract}

DOI: 10.1103/PhysRevLett.127.253001

Laser spectroscopy in combination with atomic structure calculations can be used to directly determine nuclear moments in a nuclear-theory-independent way. Such an approach is limited by the ability to calculate hyperfine structure (HFS) constants $A$ and $B$ from first principles to high precision. This problem is exacerbated in heavy atoms, where electronic correlation corrections increase. A separate problem is a determination of the accuracy of the theoretical computations, as the theory uncertainty directly contributes to the uncertainty of the extracted nuclear magnetic-dipole and electric-quadrupole moments. While numerical uncertainties can be generally determined, estimating other uncertainties is difficult, because it requires a good understanding of the relative importance of various contributions and method accuracy. In addition, theoretical computations require modeling of magnetization distribution, which is generally not known.

In this work, we consider a solution to these problems for the ${ }^{229} \mathrm{Th}$ nucleus, motivated by its unique features described below. The development of precision methods for extracting nuclear moments from laser spectroscopy measurements becomes of particular importance now, as more rare isotopes will become available with high yield at the

Published by the American Physical Society under the terms of the Creative Commons Attribution 4.0 International license. Further distribution of this work must maintain attribution to the author(s) and the published article's title, journal citation, and DOI.
Facility for Rare Isotope Beams [1] for exploring nuclear physics properties, especially of actinides.

As was established more than 40 years ago, the nuclear transition frequency between the ground and first excited states of ${ }^{229} \mathrm{Th}$ is unusually small and amounts to only several $\mathrm{eV}$ [2]. Subsequent measurements of this quantity confirmed it; the current most precise value of 8.19(12) eV [3] is an average of two recent measurements [4,5]. Such a unique feature of this isotope opens up a number of theoretical and experimental research opportunities. Special interest in this nuclear transition is motivated by a possibility to build a superprecise nuclear clock [6] and very high sensitivity to the effects of possible temporal variation of the fundamental constants, including the fine structure constant $\alpha$, strong interaction, and quark mass $[7,8]$.

The present uncertainty in the nuclear transition frequency $0.12 \mathrm{eV}$, corresponding to $\sim 30 \mathrm{THz}$, is many orders of magnitude greater than the natural linewidth, expected to be in the millihertz range. To determine the nuclear transition frequency with laser spectroscopic precision, as well as other properties of the ground and isomeric nuclear states, further experimental and theoretical investigations are required [3]. Using the experimentally measured and theoretically calculated HFS constants $A$ and $B$, the nuclear magnetic dipole and electric quadrupole moments were determined in Ref. [9] to be $\mu=0.360(7) \mu_{N}$ and $Q=$ 3.11(6) $e \mathrm{~b}$ (where $\mu_{N}$ is the nuclear magneton and $e$ is the elementary charge). This value of the magnetic moment contradicts the result $\mu=0.46(4) \mu_{N}$ found in Ref. [10] and the recent nuclear calculation value $0.43-0.48 \mu_{N}$ obtained in 
Ref. [11]. Motivated by a necessity to confirm the results of Ref. [9] and by a need to better understand the $\mathrm{Th}^{3+}$ properties for the development of the nuclear clock [3], we further developed the coupled-cluster single double triple (CCSDT) method, fully including into consideration both valence and core triple excitations, and applied it for the high-accuracy calculation of the ${ }^{229} \mathrm{Th}^{3+}$ properties. To the best of our knowledge, due to the exceptional complexity of the problem and very high computational demands, the core triples were never included in the computation of the properties of complicated atomic systems prior to this work.

The simplest version of this approach, the linearized coupled-cluster single double (LCCSD) method, was developed in Ref. [12]. In this version, only the linear terms involving the single $(S)$ and double $(D)$ excitations of the valence and core electrons were considered. A wide range of properties of univalent systems can be calculated with a very good accuracy using the LCCSD method (see, e.g., [13]). But the systematic prediction of the properties of transition matrix elements and HFS constants with an accuracy below $1 \%$ requires the inclusion of the terms beyond LCCSD, i.e., the nonlinear (NL) terms and the triple excitations. For instance, the LCCSD values of the HFS constants of the $6 s$ and $6 p_{1 / 2}$ states for ${ }^{133} \mathrm{Cs}$ differ from the experimental results by $6 \%$ [13].

Schematically single, double, and triple excitations are presented in Fig. 1. Triple core-valence [or just "valence" (vT)] terms and core terms involve excitations of two core electrons and a valence electron and three core electrons, respectively. The quadratic NL single and double terms are combinations of the single and double excitations of the electrons that take the form of $S^{2}, S D$, and $D^{2}$ combinations.

The NL and/or vT terms (in the leading order) were taken into account in a number of works [9,14-20]. A more sophisticated approach, where the equations for the valence triples were solved iteratively, was developed and applied in Refs. [21-23]. A detailed study of contributions of the
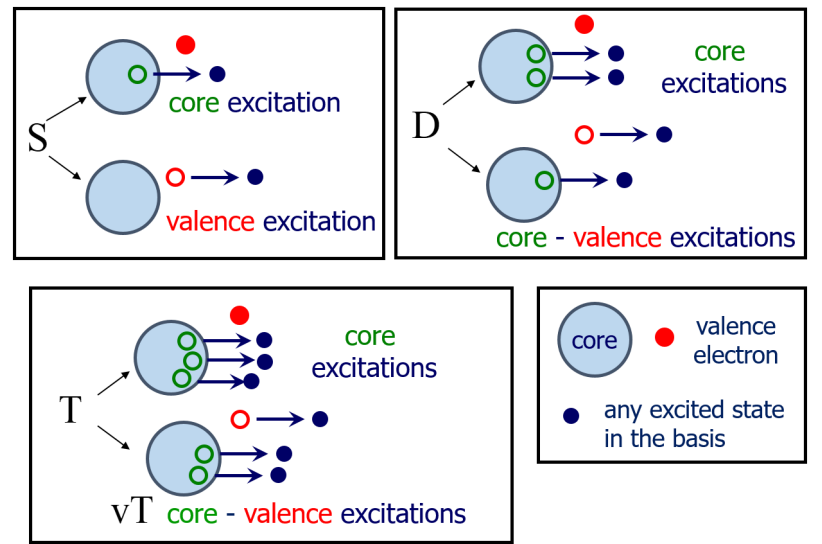

FIG. 1. Illustration of single $(S)$, double $(D)$, and triple $(T)$ excitations in the coupled-cluster approach.
$\mathrm{NL}$ and vT terms in calculating the energies, magnetic dipole HFS constants, and $E 1$ transition amplitudes in $\mathrm{Ba}^{+}$ was presented in the recent work [24], which stressed the need for inclusion of the core triple terms.

Aiming to improve the calculation accuracy of the HFS constants and, respectively, to reduce the uncertainty of $\mu$ and $Q$, we made the next step in developing the method and included the core triple excitations. The equations for the core triples were solved iteratively, using similar formalism as developed for valence triples in Ref. [21].

Method of calculation.-We evaluated the energies and HFS constants $A$ and $B$ of the lowest-lying states using a version of the high-precision relativistic coupled-cluster method developed in Ref. [21] and augmented by the inclusion of the core triple excitations. The quadratic NL terms were also included in the equations for singles and doubles but omitted in the equations for triples. The cubic and higher-order terms were also omitted as these are expected to be small.

We consider $\mathrm{Th}^{3+}$ as a univalent ion with the ground state configuration $[\mathrm{Ra}] 5 f_{5 / 2}$. The initial Dirac-HartreeFock (DHF) self-consistency procedure included the Breit interaction and was done for the core $\left[1 s^{2}, \ldots, 6 p^{6}\right]$ electrons; the $5 f$ and $6 d$ orbitals were constructed in the frozencore potential. The remaining virtual orbitals were formed using 40 basis set $B$-spline orbitals. The basis set included partial waves with the orbital quantum number up to $l=6$.

The coupled-cluster equations were solved in a basis set consisting of single-particle states. In the equations for singles, doubles, and valence triples, the sums over excited states were carried out with 35 basis orbitals with the orbital quantum number $l \leq 6$. Because of high computational demands to the iterative solution of the equations for the core triple excitations, certain restrictions were applied. We solved these equations allowing the core excitations from the $[4 d-6 p]$ core shells, the maximal orbital quantum number of all excited orbitals was equal to 5 , and the largest principal quantum number of the virtual orbitals where excitations were allowed was 25 . But, as our estimates showed, the omitted electron excitations can contribute to the removal energies of the valence states only at the level of several tens of $\mathrm{cm}^{-1}$ and can change the HFS constants at the level of $0.1 \%$. Thus, we took the triple excitations into consideration practically in full.

Energies.-Numerical results for the energies are presented in Table I. The lowest-order DHF contribution to the energies (with the inclusion of the Breit interaction) is labeled "BDHF." At the next step, we carried out the calculation in the linearized coupled-cluster single double (LCCSD) approximation. Then, we subsequently added the NL terms, valence and core triples into consideration, designating these calculations as CCSD, CCSDvT, and CCSDT, respectively. Thus, each subsequent calculation includes all terms taken into account at the previous stage and the additional terms specific for the present approximation. 
TABLE I. The removal energies of the low-lying states for $\mathrm{Th}^{3+}\left(\right.$ in $\mathrm{cm}^{-1}$ ) in different approximations, discussed in the text, are presented. The theoretical total and experimental results are given in the rows $E_{\text {total }}$ and $E_{\text {expt }}$, respectively. The difference between the total and experimental values is presented (in percent) in the row labeled "Diff. (\%)."

\begin{tabular}{lcccc}
\hline \hline & $5 f_{5 / 2}$ & $5 f_{7 / 2}$ & $6 d_{3 / 2}$ & $6 d_{5 / 2}$ \\
\hline$E_{\mathrm{BDHF}}$ & 207310 & 203393 & 211842 & 207686 \\
$E_{\mathrm{LCCSD}}$ & 232308 & 227978 & 222871 & 217543 \\
$E_{\mathrm{CCSD}}$ & 231640 & 227307 & 222490 & 217174 \\
$E_{\mathrm{CCSDvT}}$ & 230819 & 226538 & 222472 & 217259 \\
$E_{\text {CCSDT }}$ & 230693 & 226398 & 222268 & 217032 \\
$\Delta E_{\text {extrap }}$ & 1055 & 1032 & 257 & 242 \\
$E_{\text {total }}$ & 231748 & 227431 & 222526 & 217274 \\
$E_{\text {expt }}[25,26]$ & 231065 & 226740 & 221872 & 216579 \\
Diff. $(\%)$ & 0.30 & 0.30 & 0.29 & 0.32 \\
$\Delta_{\text {LCCSD }}{ }^{a}$ & 2298 & 2271 & 1256 & 1205 \\
$\Delta_{\text {CCSD }}$ & 1630 & 1599 & 875 & 836 \\
$\Delta_{\text {CCSDvT }}$ & 809 & 831 & 857 & 922 \\
$\Delta_{\text {CCSDT }}$ & 683 & 691 & 654 & 695 \\
\hline${ }^{a} \Delta_{X} \equiv E_{X}+\Delta E_{\text {extrap }}-E_{\text {expt }}$. & & &
\end{tabular}

In this way, the most complete calculation is carried out in the CCSDT approximation.

The removal energies of the valence states obtained on each stage are presented in the table. We also found complementary correction due to the basis extrapolation $\left(\Delta E_{\text {extrap }}\right)$, determined as the contribution of the higher $(l>6)$ partial waves. Based on an empiric rule obtained for Ag-like ions in Ref. [27] and used in Ref. [24], we estimate this contribution as the difference of two calculations carried out with $l_{\max }=6$ and $l_{\max }=5$. We note that this contribution is comparable with that of the triples. The total values, presented in the row labeled " $E_{\text {total }}$ " are found as $E_{\mathrm{CCSDT}}+\Delta E_{\text {extrap }}$.

For the $5 f$ and $6 d$ states, the quantum-electrodynamic corrections to the energies are small, and we did not include them in the full-scale calculation. An estimate of this effect, made following Ref. [28], shows that it can potentially change the removal energies of the considered states at the level of $100-200 \mathrm{~cm}^{-1}$.

The experimental removal energy for the ground state is $231065(200) \mathrm{cm}^{-1}$ [25]; i.e., its uncertainty is comparable to the difference between our total value and the experimental result. The experimental values for the excited states were taken from Ref. [26].

To illustrate a consistent improvement in the results when we successively add different coupled-cluster terms, we present the differences between the theoretical and experimental values obtained at each stage in the lower panel in Table I. Comparing $\Delta_{\text {CCSDT }}$ and $\Delta_{\text {LCCSD }}$, we see that the difference between the theory and experiment decreased by almost 4 times for the $5 f$ states and 2 times for the $6 d$ states when we included the NL terms and triples.
TABLE II. The theoretical and experimental [26] transition energies $\left(\right.$ in $\mathrm{cm}^{-1}$ ) of the excited states counted from the ground state.

\begin{tabular}{lrccc}
\hline \hline & Theory & Experiment & Diff. $\left(\mathrm{cm}^{-1}\right)$ & Diff. (\%) \\
\hline $5 f_{7 / 2}$ & 4318 & 4325 & 7 & 0.16 \\
$6 d_{3 / 2}$ & 9223 & 9193 & -30 & -0.33 \\
$6 d_{5 / 2}$ & 14475 & 14486 & 11 & 0.08 \\
\hline \hline
\end{tabular}

For completeness, using the total values of the removal energies, we present in Table II the theoretical transition energies counted from the ground state and compare them with the experimental data [26].

Hyperfine structure constants. - The magnetic dipole and electric quadrupole HFS constants $A$ and $B$ were calculated for the low-lying states of ${ }^{229} \mathrm{Th}^{3+}$ in Refs. [9,29]. In Ref. [9], the authors also used for the calculation the coupled-cluster method but a significantly less sophisticated version. In this work, we carry out a more complete calculation, including the NL terms and the valence and core triple excitations. In addition, our calculation is pure $a b$ initio; no semiempirical methods are applied.

The results for the magnetic-dipole HFS constants $A_{t} \equiv$ $A / g$ [where $g=\left(\mu / \mu_{N}\right) / I$ is the $g$ factor and $I$ is the nuclear spin, $I=5 / 2$ ] are presented in Table III.

The LCCSD and BDHF values and the difference between them, $\Delta(\mathrm{SD})$, are given in the upper panel of the table. Rows 4-6 give the corrections due to the NL terms, $\Delta(\mathrm{NL})$, and the valence and core triples, $\Delta(\mathrm{vT})$ and $\Delta(\mathrm{cT})$, respectively. The CCSDT values, obtained as the sum of the LCCSD values and the NL, vT, and cT

TABLE III. Different contributions to $A_{t}$ (in megahertz) and obtaining the recommended value of $g$ are explained in the text. The experimental values of the HFS constants $A$ [30] are given in the row labeled " $A$ (experim.)." The uncertainties are given in parentheses.

\begin{tabular}{lcccc}
\hline \hline & $5 f_{5 / 2}$ & $5 f_{7 / 2}$ & $6 d_{3 / 2}$ & $6 d_{5 / 2}$ \\
\hline BDHF & 507 & 263 & 831 & 304 \\
$\Delta(\mathrm{SD})$ & 72 & -45 & 268 & -386 \\
LCCSD & 579 & 218 & 1099 & -81 \\
$\Delta(\mathrm{NL})$ & -3.3 & -4.6 & -17 & 18 \\
$\Delta(\mathrm{vT})$ & -12 & -5.1 & -21 & -46 \\
$\Delta(\mathrm{cT})$ & -1.5 & -2.3 & 5.8 & -1.8 \\
CCSDT & 562 & 206 & 1067 & -111 \\
Basis extrap. & -0.2 & 2.6 & -4.5 & 7.1 \\
Total & $562(3)$ & $209(3)$ & $1063(12)$ & $-104(22)$ \\
Ref. [9] & 573 & 215 & 1079 & -92 \\
$A$ (experim.) [30] $^{\text {a }}$ & $82.2(6)$ & $31.4(7)$ & $155.3(1.2)$ & $-12.6(7)$ \\
$g($ recommended) & \multicolumn{5}{c}{$0.1465(24)$} & \\
Ref. [9] & \multicolumn{5}{c}{$0.1440(28)$} \\
\hline \hline
\end{tabular}

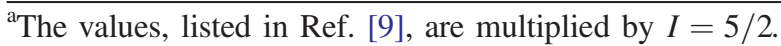


corrections, are presented in the row labeled "CCSDT." The basis extrapolation corrections are given in the row labeled "Basis extrap." The total values, listed in the row "Total," are found as the sum of the CCSDT value and the basis extrapolation correction.

Based on a comparison of the theoretical and experimental HFS constants for a number of univalent elements, the authors of Ref. [9] suggested a method to estimate the uncertainties of these constants. The uncertainty of the $A$ and $B$ calculations is expected to be on the order of $3 \%-6 \%$ of the total correlation correction (found as the difference between the final and LCCSD value), if this correction does not exceed $50 \%$. Following this approach, we estimate the uncertainties of $A_{t}$ for the $5 f_{5 / 2}, 5 f_{7 / 2}$, and $6 d_{3 / 2}$ states as $5 \%$ of their total correlation corrections. For the $6 d_{5 / 2}$ state, the correlation correction is very large, and the validity of this method is questionable. Applying it, we roughly estimate the uncertainty of $A_{t}$ for this state at the level of $20 \%-25 \%$.

We note that all values presented in Table III were obtained for the nucleus considered as the charged ball with uniform magnetization. But, as was shown in Ref. [31], the nucleus of ${ }^{229} \mathrm{Th}$ has a complex structure and different collective effects, such as quadruple-octupole vibrationrotation motion of the nucleus, the single-particle motion of the unpaired nucleon, and the Coriolis interaction between this nucleon and the nuclear core, are important. Thus, the real nuclear magnetization can differ from the uniform magnetization. To investigate this problem, we follow the approach developed in Ref. [32]. We can express the HFS constant $A$ as

$$
A=g A_{0}\left(1-d_{\text {nuc }} y\right)
$$

where $A_{0}$ is the theoretical value calculated at the pointlike magnetization of the nucleus and $d_{\text {nuc }}$ and $y$ are the parameters depending on the nuclear and electronic structure, respectively. The quantities $g$ and $d_{\text {nuc }}$ are assumed to be unknown. The experimental value of $A$ can be used on the left-hand side of Eq. (1), and $y$ can be found from the calculation.

Indeed, taking into account that $d_{\text {nuc }}=0$ and $d_{\text {nuc }}=1$ correspond to the pointlike and uniform magnetization, respectively, we can find $y$ from Eq. (1) as

$$
y=1-A_{t} / A_{0},
$$

where $A_{t}$ are given in Table III for different states. We note that the ratios $A_{t} / A_{0}$ are very stable and insensitive to different corrections, and we determine the uncertainty of $y$ at the level of $0.02 \%$.

To find $g$ and $d_{\text {nuc }}$, we use the HFS constants for the $5 f_{5 / 2}$ and $6 d_{3 / 2}$ states, which are known most precisely both experimentally and theoretically. Using for each of them Eq. (1) and solving the system of two equations in two unknowns, we arrive at

$$
\begin{aligned}
& g \approx 0.1465(24), \\
& d_{\mathrm{nuc}} \approx 1.7(2.1) .
\end{aligned}
$$

Thus, the $g$ factor is determined with the accuracy $\sim 1.5 \%$. Using this value, we find the nuclear magnetic moment: $\mu_{I}=g I \approx 0.366(6)$. This result is in good agreement with that reported in Ref. [9]: $\mu_{I} \approx 0.360(7)$. We note that the uncertainty estimate in Ref. [9] did not include uncertainty due to the magnetization distribution.

For heavy nuclei, the parameter $d_{\text {nuc }}$ can vary over a wide range. For example, for the gold isotopes ${ }^{197,193,191} \mathrm{Au}$ with the nuclear spin of $3 / 2, d_{\text {nuc }}=-5.5(6)$ [32]. Our result (4) suggests that for ${ }^{229} \mathrm{Th}$ the absolute value of $d_{\text {nuc }}$ is smaller and it is most likely positive. As a result, the correction to the $g$ factor, due to inhomogeneity of the nuclear magnetization, is small but not negligible.

In Table IV, we present the results obtained for the electric-quadrupole HFS constants $B / Q$. All designations in the upper panel of the table are the same as in Table III. In the lower panel, we present the experimental results for the HFS constants $B$ [30]. The values of $Q$ (in $e$ b) are found as the ratios of $B$ (experim.) and values listed in the row labeled "Total."

The values of $B / Q$ obtained in this work turned out to be between the results of Refs. $[9,29]$ but are somewhat closer to the former. The experimental uncertainty for

TABLE IV. Different contributions to the electric-quadrupole HFS constants $B / Q$ [in MHz/(eb))] for ${ }^{229} \mathrm{Th}^{3+}$, explained in the text, are presented. The experimental values of the HFS constants $B$ [30] are given in the row labeled " $B$ (experim.)." The values of $Q$ (in $e$ b) are obtained as the ratios of $B$ (experim.) and values listed in the row labeled "Total"; the recommended value of $Q$ (in $e$ b) is given in the row labeled " $Q$ (recommended)." The uncertainties are given in parentheses.

\begin{tabular}{lcccc}
\hline \hline & $5 f_{5 / 2}$ & $5 f_{7 / 2}$ & $6 d_{3 / 2}$ & $6 d_{5 / 2}$ \\
\hline BDHF & 535 & 572 & 611 & 648 \\
$\Delta($ SD $)$ & 202 & 251 & 132 & 228 \\
LCCSD & 737 & 822 & 743 & 877 \\
$\Delta($ NL $)$ & 38 & 45 & 9 & 9 \\
$\Delta($ vT $)$ & -55 & -57 & -34 & -27 \\
$\Delta(\mathrm{cT})$ & 3 & 3 & 7 & 7 \\
CCSDT & 723 & 814 & 725 & 866 \\
Basis extrap. & 6 & 8 & 3 & 4 \\
Total & $729(10)$ & $822(13)$ & $728(6)$ & $869(11)$ \\
Ref. [9] & 725 & 809 & 738 & 873 \\
Ref. [29] & 740 & 860 & 690 & 860 \\
$B$ (experim.) [30] & $2269(2)$ & $2550(12)$ & $2265(9)$ & $2694(7)$ \\
$Q$ & $3.11(4)$ & $3.10(5)$ & $3.11(3)$ & $3.10(4)$ \\
$Q$ (recommended) & \multicolumn{5}{c}{$3.11(2)$} \\
Ref. [9] & \multicolumn{5}{c}{$3.11(6)$} \\
Refs. [29,30] & \multicolumn{5}{c}{ a } & \\
\hline \hline
\end{tabular}

${ }^{\mathrm{a}}$ This result was obtained using the measurements of Ref. [30] and calculations of Ref. [29]. 
the constants $B$ does not exceed $0.5 \%$, while the theoretical uncertainties are at the level of $0.8 \%-1.5 \%$. The uncertainties were determined in the same manner as was done for the HFS constants $A$. The constants $B$ are large for all four considered states, and their fractional uncertainties are comparable. For this reason, the recommended value was obtained as the weighted average over four values of $Q$ given in Table IV. We note the perfect agreement of our recommended value with the results obtained in Refs. $[9,29,30]$, but our uncertainty is a few times smaller.

Conclusion.-We have developed the relativistic CCSDT method with the full inclusion of valence and core linear triple excitations. Using the theoretical values of the HFS constants obtained in this work and the experimental results [9], we determined the values of the nuclear magnetic dipole and electric quadrupole moments. Analyzing the results obtained at the different stages, we determined the uncertainties of the recommended values to be $1.5 \%$ for $\mu_{I}$ and $0.6 \%$ for $Q$. We investigated the effect of the inhomogeneity of the nuclear magnetization and found it to be small but not negligible. We conclude that it should be taken into account to determine the magnetic dipole nuclear moment $\mu_{I}$ with accuracy better than $1 \%$. Further experimental work to measure the HFS (especially magnetic dipole) constants more precisely is needed to improve precision and better understand the effect of nuclear magnetization. The method developed in this work can be used for any monovalent system and, when combined with configuration interaction, can be extended to systems with 2-6 valence electrons.

We are grateful to Yu. Demidov for useful discussion. This work is a part of the "Thorium Nuclear Clock" project that has received funding from the European Research Council (ERC) under the European Union's Horizon 2020 research and innovation program (Grant Agreement No. 856415). S. G. P. and M. G. K. acknowledge support by the Russian Science Foundation under Grant No. 19-1200157. This research was supported in part through the use of UDEL Caviness and DARWIN computing systems: DARWIN-A Resource for Computational and Dataintensive Research at the University of Delaware and in the Delaware Region, Rudolf Eigenmann, Benjamin E. Bagozzi, Arthi Jayaraman, William Totten, and Cathy H. $\mathrm{Wu}$, University of Delaware.

[1] E. P. Abel et al., J. Phys. G 46, 100501 (2019).

[2] L. A. Kroger and C. W. Reich, Nucl. Phys. A259, 29 (1976).

[3] E. Peik, T. Schumm, M. Safronova, A. Pálffy, J. Weitenberg, and P. Thirolf, Quantum. Sci. Tech. 6, 034002 (2021).

[4] B. Seiferle, L. von der Wense, P. V. Bilous, I. Amersdorffer, C. Lemell, F. Libisch, S. Stellmer, T. Schumm, C. E. Düllmann, A. Pálffy, and P. G. Thirolf, Nature (London) 573, 243 (2019).
[5] T. Sikorsky, J. Geist, D. Hengstler, S. Kempf, L. Gastaldo, C. Enss, C. Mokry, J. Runke, C. E. Düllmann, P. Wobrauschek, K. Beeks, V. Rosecker, J. H. Sterba, G. Kazakov, T. Schumm, and A. Fleischmann, Phys. Rev. Lett. 125, 142503 (2020).

[6] E. Peik and C. Tamm, Europhys. Lett. 61, 181 (2003).

[7] V. V. Flambaum, Phys. Rev. Lett. 97, 092502 (2006).

[8] P. Fadeev, J. C. Berengut, and V. V. Flambaum, Phys. Rev. A 102, 052833 (2020).

[9] M. S. Safronova, U. I. Safronova, A. G. Radnaev, C. J. Campbell, and A. Kuzmich, Phys. Rev. A 88, 060501(R) (2013).

[10] S. Gerstenkorn, P. Lue, J. Verges, D. W. Englekemeir, J. E. Gindler, and F. S. Tomkins, J. Phys. (Paris) 35, 483 (1974).

[11] N. Minkov and A. Pálffy, Phys. Rev. C 103, 014313 (2021).

[12] S. A. Blundell, W. R. Johnson, and J. Sapirstein, Phys. Rev. A 43, 3407 (1991).

[13] M. S. Safronova and W. R. Johnson, Adv. At. Mol. Opt. Phys. 55, 191 (2008).

[14] S. Salomonson and P. Öster, Phys. Rev. A 41, 4670 (1990).

[15] S. Salomonson and A. Ynnerman, Phys. Rev. A 43, 88 (1991).

[16] E. Eliav, U. Kaldor, and Y. Ishikawa, Phys. Rev. A 53, 3050 (1996).

[17] R. Pal, M. S. Safronova, W. R. Johnson, A. Derevianko, and S. G. Porsev, Phys. Rev. A 75, 042515 (2007).

[18] K. J. Arnold, S. R. Chanu, R. Kaewuam, T. R. Tan, L. Yeo, Z. Zhang, M. S. Safronova, and M. D. Barrett, Phys. Rev. A 100, 032503 (2019).

[19] B. K. Sahoo, Phys. Rev. A 74, 020501(R) (2006).

[20] B. K. Sahoo, B. P. Das, R. K. Chaudhuri, and D. Mukherjee, Phys. Rev. A 75, 032507 (2007).

[21] S. G. Porsev and A. Derevianko, Phys. Rev. A 73, 012501 (2006).

[22] S. G. Porsev, K. Beloy, and A. Derevianko, Phys. Rev. Lett. 102, 181601 (2009).

[23] S. G. Porsev, K. Beloy, and A. Derevianko, Phys. Rev. D 82, 036008 (2010).

[24] S. G. Porsev and M. S. Safronova, Phys. Rev. A 103, 042815 (2021).

[25] Yu. Ralchenko, A. Kramida, J. Reader, and the NIST ASD Team, NIST Atomic Spectra Database (version 4.1) (2011), available at http://physics.nist.gov/asd. National Institute of Standards and Technology, Gaithersburg, MD.

[26] http://www.lac.u-psud.fr/old-lac/lac/Database/Tab-energy/ Thorium/Th-el-dir.html.

[27] M. S. Safronova, V. A. Dzuba, V. V. Flambaum, U. I. Safronova, S. G. Porsev, and M. G. Kozlov, Phys. Rev. A 90, 042513 (2014).

[28] I. I. Tupitsyn, M. G. Kozlov, M. S. Safronova, V. M. Shabaev, and V. A. Dzuba, Phys. Rev. Lett. 117, 253001 (2016).

[29] J. C. Berengut, V. A. Dzuba, V. V. Flambaum, and S. G. Porsev, Phys. Rev. Lett. 102, 210801 (2009).

[30] C. J. Campbell, A. G. Radnaev, and A. Kuzmich, Phys. Rev. Lett. 106, 223001 (2011).

[31] N. Minkov and A. Pálffy, Phys. Rev. Lett. 118, 212501 (2017).

[32] Yu. A. Demidov, E. A. Konovalova, R. T. Imanbaeva, M. G. Kozlov, and A.E. Barzakh, Phys. Rev. A 103, 032824 (2021). 